\title{
Electron- and phonon-mediated ultrafast magnetization dynamics of $\mathrm{Gd}(0001)$
}

\author{
Muhammad Sultan, ${ }^{1,2}$ Unai Atxitia, ${ }^{3}$ Alexey Melnikov, ${ }^{1,4}$ Oksana Chubykalo-Fesenko, ${ }^{3}$ and Uwe Bovensiepen ${ }^{2, *}$ \\ ${ }^{1}$ Fachbereich Physik, Freie Universität Berlin, Arnimallee 14, 14195 Berlin, Germany \\ ${ }^{2}$ Fakultät für Physik and Center for Nanointegration (CeNIDE), Universität Duisburg-Essen, Lotharstrasse 1, 47048 Duisburg, Germany \\ ${ }^{3}$ Instituto de Ciencia de Materiales de Madrid, CSIC, Cantoblanco, 28049 Madrid, Spain \\ ${ }^{4}$ Fritz-Haber-Institut der Max-Planck Gesellschaft, Faradayweg 4-6, 14195 Berlin, Germany \\ (Received 1 June 2011; revised manuscript received 9 March 2012; published 8 May 2012)
}

\begin{abstract}
Here we report on the ultrafast magnetization dynamics of $\mathrm{Gd}(0001)$, which we investigated as a function of equilibrium temperature by employing the femtosecond time-resolved magneto-optical Kerr effect (MOKE) and modeling by the Landau-Lifshitz-Bloch equation in combination with the two-temperature model. Based on the observed temperature-dependent transient MOKE signals, we separate the magnetization dynamics into two regimes at delays of (i) a few picoseconds and (ii) several 100 femtoseconds. In the picosecond regime, the demagnetization time determined from the experiment increases with temperature from $0.8 \mathrm{ps}$ at $50 \mathrm{~K}$ to $1.5 \mathrm{ps}$ at $280 \mathrm{~K}$. A successful description of this observation was achieved by considering the dynamics of the $4 f$ spin system coupled to $5 d$ conduction electrons within two coupling mechanisms: (a) through electronic scattering and (b) spin-flip scattering mediated by phonons. We conclude that at temperatures below the Debye temperature, a hot electron-mediated process describes the experimentally found demagnetization times of $\approx 0.8 \mathrm{ps}$ well. At higher temperatures phonon-mediated processes have to be included to explain the 2 times longer demagnetization time. In the second regime at time delays of few $100 \mathrm{fs}$ we find an increase in the MOKE rotation and ellipticity at $50 \mathrm{~K}$ at delays before demagnetization sets in. Above $50 \mathrm{~K}$ the transient changes in rotation and ellipticity are of opposite sign. We explain this behavior by competing magnetic and nonmagnetic contributions in the transient MOKE signals at these delays directly after optical excitation when excited phonons do not yet facilitate angular momentum transfer to the lattice.
\end{abstract}

DOI: 10.1103/PhysRevB.85.184407

PACS number(s): 75.78.Jp, 75.40.Gb, 78.20.Ls

\section{INTRODUCTION}

Routine access to ultrafast time scales by means of femtosecond (fs) laser sources has in recent years pushed the experimentally reachable magnetization dynamics into the highly nonequilibrium regime. Under such excitation conditions the transient distribution functions of subsystems like charge, lattice, spin, and orbital moments are not in equilibrium with each other and a wide range of novel phenomena are currently being reported. ${ }^{1}$ Prominent examples are laser-driven formation of ferromagnetic order ${ }^{2,3}$ and magnetization reversal by individual laser pulses. ${ }^{4}$ Both demonstrate the potential for spin manipulation in solid materials on ultrafast time scales and are promising for future applications in ultrafast memory devices. The search for the appropriate combinations of materials and excitation conditions ${ }^{5}$ can be considered as a top-down approach toward ultrafast magnetic applications. The goal to obtain microscopic insight into the elementary processes and interactions that couple excitations like electronhole pairs, magnons, and phonons can be viewed as the complementary bottom-up approach. It is interesting to note that a controllable all-optical magnetization switching has been until now reported only for a combination of transition and rare earth metals such as $\mathrm{FeCoGd} .^{5}$

The current literature on ultrafast magnetization dynamics is controversial since the process responsible for the magnetization reduction after femtosecond laser excitation is still ambiguous. Several contributions are currently under debate: ${ }^{6,7}$ (i) phonon-assisted spin flips [phonon-mediated Elliott-Yafet (EY) processes] that transfer angular momentum from the magnetization to the lattice are considered in a microscopic three-temperature model $(\mathrm{M} 3 \mathrm{TM}){ }^{8}$ (ii) hot electron mediated spin flips at "hot spots" of the electronic band structure originating from spin-orbit interaction ${ }^{9-12}$ and electron-electron EY scattering: ${ }^{13}$ and (iii) laser-induced transport processes that essentially drive spin polarization of the conduction electrons. ${ }^{14,15}$ The spin system also contributes to the observed demagnetization rates, slowing them down at elevated temperatures $T \rightarrow T_{C}$ via the spin-spin interaction and critical slowing-down effects. ${ }^{16,17}$ An obvious important question is which mechanisms act in which materials and to what degree these processes act simultaneously or compete with each other. While in transition metals it has been argued that the electronic mechanism may suffice to describe the ultrafast demagnetization, ${ }^{11,13}$ in other materials this is an open question.

The rare-earth metal $\mathrm{Gd}$ is an important example of the diversity of different materials since its response to femtosecond laser excitation has been experimentally reported to be slower than in transition metals. ${ }^{18,19}$ Several mechanisms for ultrafast demagnetization in Gd have been discussed on theoretical background: the EY phonon-mediated scattering, ${ }^{8}$ the diffusion mechanism, ${ }^{14}$ and the direct spin-phonon interaction caused by the spin-orbit coupling such as the Raman processes. ${ }^{20}$ However, the experimental results suggest that the scattering of conduction-band electrons is also relevant. ${ }^{19}$ The predicted time scale associated with the Raman processes of the order of $100 \mathrm{ps}$ does not account for all observed demagnetization at the femto- and picosecond range. Here we aim at a combined theoretical and experimental study with systematically changing experimental conditions to elucidate this problem.

The magnetic moment of $\mathrm{Gd}$ consists of $7 \mu_{\mathrm{B}}$ originating from the half-filled $4 f$ shell and $0.58 \mu_{\mathrm{B}}$ from the $5 d$ 
conduction band that is generated through intra-atomic exchange coupling $J_{4 f-5 d} \cdot{ }^{21,22}$ Albeit the total magnetization of $\mathrm{Gd}$ in equilibrium $M$, i.e., on infinitely long time scales, is the sum of these $5 d$ and $4 f$ contributions, a dynamic picture might require a separate treatment of the localized and the conduction electrons. In the model that we employ here to describe the laser-induced magnetization dynamics, the dynamic magnetization $\tilde{M}$ represents the localized electrons. In principle, it is reasonable to consider two distinct time intervals for the dynamics of the $4 f$ and $5 d$ magnetic moments. In one temporal interval, the relative evolution of $4 f$ and $5 d$ magnetic moments deviates from each other. In the second regime, both magnetic moments are well aligned with each other and present an identical relative variation. Due to the much larger $4 f$ contributions, this state might be dominated by the $4 f$ magnetic moment. The conduction electrons are responsible for the optical properties, mediate the exchange interaction among neighboring localized magnetic moments, and facilitate their coupling to the lattice by means of the spin-orbit interaction. Absorption of optical photons by $5 d$ electrons leads to $e$ - $h$ pair excitation in the conduction band. Excitations of $4 f$ electrons can be considered like those in an atom; they follow selection rules and require ultraviolet or $\mathrm{x}$-ray photons not used here. ${ }^{19}$ Due to the half-filled $4 f$ shell (orbital quantum number $L=0$, following Hund's rules) and hence the absence of direct spin-lattice coupling, the localized electrons respond to the optical excitation through their interaction with the conduction-band electrons only.

To discern the dynamics of the $4 f$ and $5 d$ subsystems, techniques which separate or specifically probe these subsystems are beneficial. In a recent experiment we have determined the time scale at which a change of the $4 f$ magnetic moment proceeds by femtosecond time-resolved x-ray magnetic circular dichroism (fs-XMCD) at the $M_{5}$ absorption edge. ${ }^{19}$ It was observed that Gd shows two characteristic demagnetization times following femtosecond laser excitation. First, a time scale of $0.8 \mathrm{ps}$ linked to the electron-phonon equilibration and second, a 40-ps scale which indicates quasiequilibrium spin-lattice relaxation. A magneto-optical technique should be sensitive to the magnetic moment of the $5 d$ electrons, which, however, interact with the $4 f$ magnetic moments. On ultrafast time scales nonmagnetic contributions must be considered in addition. $^{23-25}$

In this article we report the obtained femtosecond timeresolved magneto-optical Kerr effect (tr-MOKE) data for $\operatorname{Gd}(0001)$ and separate the $4 f$ and $5 d$ contributions. On this basis we are able to study the influence of the equilibrium temperature $T_{0}$ on the demagnetization dynamics. The comparison with the theoretical description allows a quantification of phonon- and electron-mediated contributions to the femtosecond-laser-induced demagnetization. We find that at temperatures well below the Debye temperature $\Theta_{D}$, electronic scattering can explain the observed demagnetization times. Above $\Theta_{D}$, phonon-mediated processes also have to be taken into account.

\section{EXPERIMENTAL RESULTS AND ANALYSIS}

We combined epitaxial film growth of $\mathrm{Gd}(0001)$ on W(110) (Refs. 26,27) in a UHV chamber with a femtosecond time-

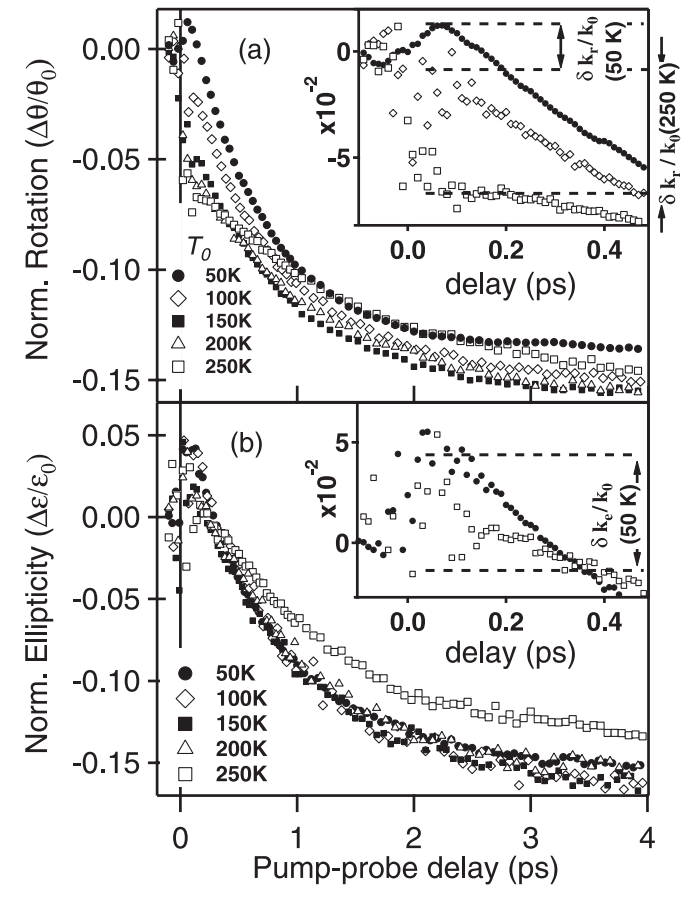

FIG. 1. Time-dependent changes of the MOKE signals after femtosecond laser excitation normalized to the respective equilibrium values for $20 \mathrm{~nm} \mathrm{Gd(0001)/W(110)} \mathrm{measured} \mathrm{at} \mathrm{different} \mathrm{equilibrium}$ temperatures $T_{0}$. Panel (a) depicts the transient MOKE rotation and (b) the MOKE ellipticity. As emphasized by the inset, rotation and ellipticity results show a different behavior within the initial few hundred femtoseconds.

resolved pump-probe experiment. Here we investigate films with a thickness of $20 \mathrm{~nm}$. The employed cavity dumped Ti:sapphire oscillator delivers 40-nJ laser pulses at 35-fs pulse duration and 800-nm wavelength at a repetition rate of $1.52 \mathrm{MHz}$. The MOKE polarization rotation $\theta$ and ellipticity $\varepsilon$, which are proportional to the magnetization under equilibrium conditions, were measured as a function of pump-probe delay $t$ as detailed in Ref. 28. In brief, in the longitudinal MOKE geometry the pump-induced change in the rotation $\Delta \theta(t)$ was recorded with an absorbed pump fluence of about $1 \mathrm{~mJ} / \mathrm{cm}^{2}$ and in an applied magnetic field of $\sim 300 \mathrm{Oe}$. Inserting a quarter wave plate allows detection of $\varepsilon$ and its pump-induced change $\Delta \varepsilon(t)$. The equilibrium temperature $T_{0}$ was varied from 50 to $280 \mathrm{~K}$ by cooling the sample with liquid $\mathrm{He}$ and then stabilizing $T_{0}$ by resistive heating at the desired value. $T_{0}$ was measured using a WRe thermocouple attached to the W(110) substrate.

Figure 1(a) shows the time dependence of the transient MOKE rotation signal $\Delta \theta$ normalized to the MOKE rotation without optical excitation $\theta_{0}$ for different $T_{0}$. We observe a pronounced variation in $\Delta \theta / \theta_{0}$ of $12-15 \%$ at a delay of $4 \mathrm{ps}$. In more detail, we recognize an initial, steplike contribution, which we term $\delta k_{r}$. This effect occurs essentially within 300 fs and is characterized by a change in the sign from positive to negative with increasing $T_{0}$, as highlighted in the inset. Subsequent to these initial effects a continuous reduction in $\Delta \theta / \theta_{0}$ was observed, which tends to saturate at $3 \mathrm{ps}$. At later time delays the signal is reduced further up to a few hundred picoseconds, ${ }^{19,28}$ which is not depicted here. Results for the MOKE ellipticity $\Delta \varepsilon(t) / \varepsilon_{0}$ are shown in Fig. 1(b). We 
recognize a similar behavior as in $\Delta \theta$ at $T_{0}<100 \mathrm{~K}$, including the $15 \%$ change at 4 ps and a change $\delta k_{e}$ at $t<300$ fs. However, with increasing $T_{0}$ the sign of $\delta k_{e}$ does not change, contrary to $\delta k_{r}$. From the temperature-dependent behavior depicted in Fig. 1, we conclude that the dynamics can be divided into two regimes. The first one at delays close to 100 fs shows a different temperature dependence for rotation and ellipticity. The second regime is characterized by an exponential decay in both MOKE signals with a characteristic time scale of about 1 ps.

Due to the optical wavelength employed in the experiment, MOKE is primarily sensitive to the conduction electrons and could contain nonmagnetic contributions. ${ }^{23-25}$ In order to determine $\tilde{M}(t)$, which was established by fs-XMCD to follow a single exponential evolution, ${ }^{19}$ from the MOKE data we assume a time-dependent factor $k_{r, e}(t)$ that accounts for the deviation from a well-defined $4 f$ magnetization dynamics in the MOKE rotation and ellipticity:

$$
\begin{aligned}
& \Delta \theta(t) / \theta_{0}=\left(\Delta \tilde{M} / \tilde{M}_{0}+1\right) \cdot k_{r}(t) / k_{0 r}-1, \\
& \Delta \varepsilon(t) / \varepsilon_{0}=\left(\Delta \tilde{M} / \tilde{M}_{0}+1\right) \cdot k_{e}(t) / k_{0 e}-1 .
\end{aligned}
$$

The determination of $\Delta \tilde{M}$ requires assumptions on $k_{r, e}(t)$. We use a linear expansion of this quantity with the transient electronic energy density $E_{e}(t)$ and show below that this is indeed sufficient to separate $\Delta \tilde{M}$ and $k_{r, e}$. This is reasonable because we observed a pronounced difference in $\Delta \theta / \theta_{0}$ and $\Delta \varepsilon / \varepsilon_{0}$ for $t \lesssim 300 \mathrm{fs}$, where the conduction electrons carry the dominant part of the excess energy. ${ }^{27,29} \mathrm{We}$ expand $k_{r, e}$ following

$$
k_{r, e}\left(T_{0}, t\right)=k_{0 r, e}\left(T_{0}\right)+\frac{\delta k_{r, e}}{\delta E_{e}} \Delta E_{e}\left(T_{0}, t\right)+\cdots
$$

The first term $k_{0}$ represents the value without laser excitation, obtained from static MOKE measurements, $\delta k_{r, e}$ is the extremum value found near $80 \mathrm{fs}$, indicated in the inset of Fig. 1, and $\delta E_{e}$ is the corresponding maximum in the electronic excess energy density $E_{e}(t)$. Assuming an electronic temperature $T_{e}$, the electronic energy density $E_{e}(t)$ is related to $T_{e}$ through $E_{e}=\gamma T_{e}(t)^{2}$, with $\gamma$ being the linear parameter in the temperature-dependent specific heat of the electron system. Here we used the calculated values of $E_{e}(t)$, employing the well-known two-temperature model $(2 \mathrm{TM})^{30}$ in a refined form with its application for $\mathrm{Gd}(0001) .{ }^{27,29}$ The parameters of the 2TM were used similar to that of Ref. 27: the $e$-ph coupling is modeled with an energy-transfer rate $H\left(T_{e}, T_{p h}\right)$, assuming thermal electron and phonon distributions; the temperature dependence of the specific heat $C_{l}$ is described using the Debye approximation with a high temperature value $C_{l}(T \rightarrow \infty)=40 \mathrm{~J} \mathrm{~mol}^{-1} \mathrm{~K}^{-1}$; thermal conductivity $\kappa$ is taken as temperature-dependent $\kappa\left(T_{e}\right)=\kappa_{0} T_{e} / T_{p h}$ with $\kappa_{0}=$ $11 \mathrm{Wm}^{-1} \mathrm{~K}^{-1} ; \gamma=225 \mathrm{~J} \mathrm{~m}^{-3} \mathrm{~K}^{-2}$; and an optical penetration depth of $20 \mathrm{~nm}$. All these parameters are not arbitrary, but the electronic dynamics calculated by the $2 \mathrm{~T}$ model was carefully compared with the photoemission experiment. Note that the 2TM is certainly a simplification of the electronic and lattice dynamics excited by the laser pulse. We follow this model here because (a) it was demonstrated ${ }^{27,29}$ to hold after $\sim 150$ fs and (b) it works well for the purpose of separation of $\tilde{M}$ and $k_{r, e}\left(T_{0}, t\right)$ here. After the separation of $\tilde{M}$ and $k_{r, e}\left(T_{0}, t\right)$, we are now able to discuss each of these quantities independently.

\section{A. Regime of several $\mathbf{1 0 0} \mathrm{fs}$}

The potential origin of the observed fast temperaturedependent contributions described here by the factors $k_{r, e} / k_{0 r, e}$ could be the temperature-dependent changes in the electronic band structure, ${ }^{31,32}$ which affect the magnetic moment of the $5 d$ electrons, and/or changes in the magneto-optical constants. In a femtosecond MOKE experiment, these contributions are difficult to disentangle and a full treatment might require future, especially theoretical work similar to Ref. 25. However, here we briefly report first observations, which we find worth noting. Following the usual caveat that such contributions could originate from optical artifacts, we first consider $k_{r, e} / k_{0 r}$ in Eq. (1) as spectroscopic factors describing the efficiency of the magneto-optical detection, which can be modified by the pump laser excitation. The temporal evolution of $k_{r, e}\left(T_{0}, t\right)$ was calculated from the time-dependent $\Delta E_{e}(t)=E_{e}(t)-E_{0}$, $E_{0}$ being the energy density at $T_{0}$ before optical excitation, from the 2TM. The results were scaled by $\delta k_{r, e}\left(T_{0}\right)$, which were determined from the experimental results as shown in the inset of Fig. 1. The resulting time evolutions of the $k_{r} / k_{0 r}$ and $k_{e} / k_{0 e}$ are shown in Figs. 2(a) and 2(b), respectively. This figure emphasizes the changes within a few hundred femtoseconds after optical excitation and their

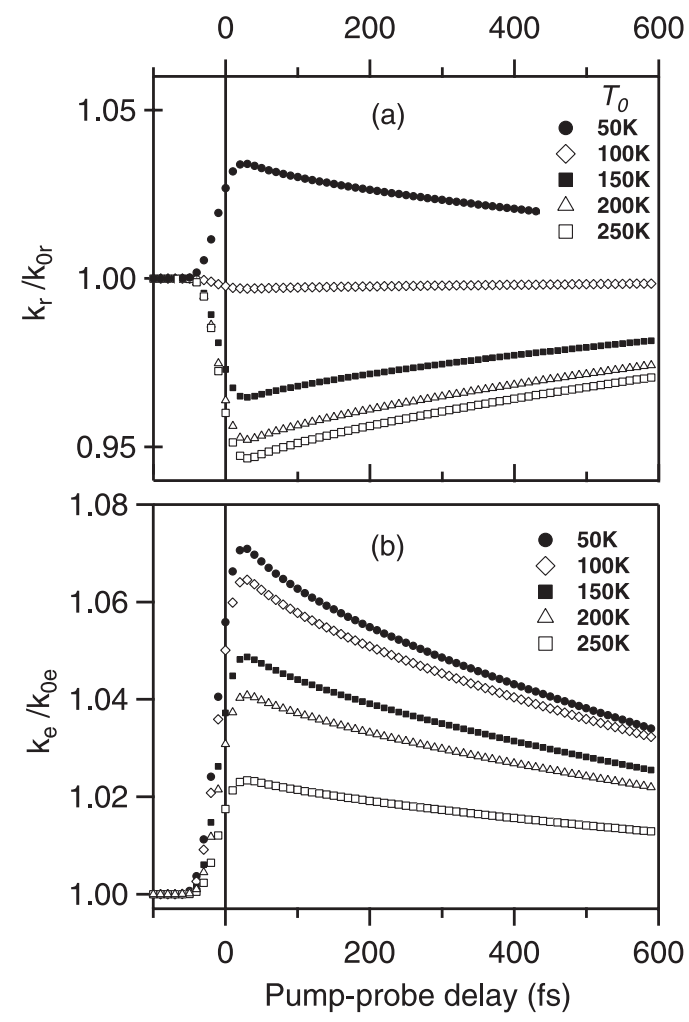

FIG. 2. The variation of $k_{r} / k_{0 r}$ and $k_{e} / k_{0 e}$ for different temperatures $T_{0}$ as a function of time. The amplitude was extracted from experimental data, and the transient evolution was calculated by the electron energy density (see text). Both $k_{r} / k_{0 r}$ and $k_{e} / k_{0 e}$ increase for $t<300 \mathrm{fs}$ at $50 \mathrm{~K}$, while at higher temperatures their respective changes are of opposite sign. 
different temperature dependencies. As shown in Fig. 2, at $T_{0}=50 \mathrm{~K}$, both $k_{r} / k_{0 r}$ and $k_{e} / k_{0 e}$ increase within $100 \mathrm{fs}$ and decrease at later delays. At higher $T_{0}, k_{r} / k_{0 r}$ and $k_{e} / k_{0 e}$ present a different behavior. While $k_{r} / k_{0 r}$ decreases to $-6 \%$, $k_{e} / k_{0 e}$ remains positive before both signals approach unity at later delays. In the following, this model is used to determine the transient $\Delta \tilde{M} / \tilde{M}_{0}$ leading to a self-consistent picture.

On the other hand, pump-induced variations of the magnetic moment of the $5 d$ electrons could indeed also contribute to the observed transient $k_{r, e} / k_{0 r, e}$, providing similar behavior. A possible scenario for such magnetic contributions is given in the outlook section. Therefore, we emphasize here that at the moment we are not able to attribute the observed behavior of $k_{r, e} / k_{0 r, e}$ unambiguously to magnetic or optical effects and leave this question open for further investigations. However, this (i) does not diminish the significance of the experimental observation itself, and (ii) it does not question the analysis of the temperature-dependent $\Delta \tilde{M} / \tilde{M}_{0}$ performed below and the resulting conclusions, as discussed at the end of the next section.

In our earlier fs-XMCD work we have shown that the $4 f$ magnetic moment changes with a time constant of 0.8 ps after laser excitation. ${ }^{19}$ We show below that this time constant is also observed in time-dependent MOKE, and our earlier work is significantly extended here by a systematic temperature-dependent study. We return to this point below in the Discussion section.

\section{B. Regime of a few picoseconds}

After several $100 \mathrm{fs}$ we find a continuous decrease of the MOKE ellipticity and the rotation, which agrees reasonably well with the dynamics of the $4 f$ magnetic moment reported in Ref. 19. We therefore discuss here the time evolution of $\tilde{M}$. In the remaining part of this paper we restrict ourself to the analysis of the magnetization dynamics at delays later than few hundred femtoseconds. We consider that at this time scale the $5 d$ and $4 f$ spin systems are in equilibrium with each other and present identical dynamics.

The transient $\Delta \tilde{M} / \tilde{M}_{0}$ was determined from $\Delta \theta / \theta_{0}$ and $\Delta \varepsilon / \varepsilon_{0}$ using Eqs. (1) and (2), as discussed in the previous section. Two resulting exemplary data sets for $\Delta \tilde{M} / \tilde{M}_{0}$, which were determined from the MOKE rotation and ellipticity, agree with each other and are depicted in the inset of Fig. 3 at $T_{0}=$ $200 \mathrm{~K}$. To illustrate the dependence on $T_{0}$, data for $\Delta \tilde{M}(t) / \tilde{M}_{0}$ are shown in the main panel of Fig. 3 after their normalization to -1 at $t=4 \mathrm{ps}$. It is evident from this comparison that the demagnetization develops more slowly for higher $T_{0}$. We determined the demagnetization time $\tau_{m}$ by fitting $\Delta \tilde{M} / \tilde{M}_{0}$ for different $T_{0}$ by a single exponential function in the time interval from 0.2 to $4 \mathrm{ps}$ and averaging over the values obtained from the MOKE rotation and ellipticity. The resulting fits are shown by the solid lines in Fig. 3. Figure 4 depicts the temperaturedependent $\tau_{m}$, which we determined by these fits, by open circles. We observe a substantial increase in $\tau_{m}$ with rising $T_{0}$. More precisely, $\tau_{m}$ exhibits a five time increase in $\partial \tau_{m} / \partial T_{0}$ at temperatures above $170 \mathrm{~K}$ compared to lower $T_{0}$. Please note that the original experimental data of Fig. 1 was also fitted for comparison. This analysis yields an offset in $\tau_{m}$ of $150 \mathrm{fs}$. However, the overall temperature-dependent change in $\tau_{m}$ was

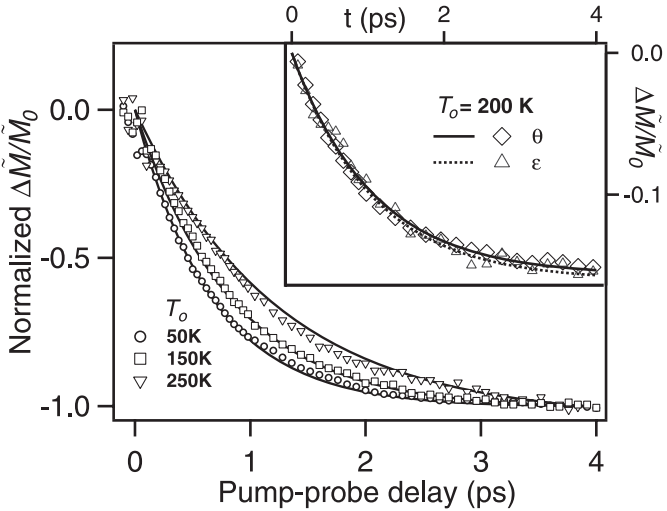

FIG. 3. The main panel depicts $\Delta \tilde{M} / \tilde{M}_{0}$ extracted from $\Delta \theta / \theta_{0}$ for selected equilibrium temperatures. The curves have been normalized to -1 at $t=4 \mathrm{ps}$. The inset compares the transient changes in the magnetization $\Delta \tilde{M} / \tilde{M}_{0}$ at $T_{0}=200 \mathrm{~K}$ which were determined from the MOKE rotation and the ellipticity measurements. Lines represent single exponential fits.

comparable to the results reported in Fig. 4, and we conclude that our analysis is robust regarding the temperature-dependent change in $\tau_{m}$.

To understand the underlying mechanism of the demagnetization, we employ theoretical modeling of the demagnetization using the Landau-Lifshitz-Bloch (LLB) equation, which is discussed in the following section.

\section{THEORETICAL MODELING BY THE LANDAU-LIFSHITZ-BLOCH EQUATION}

The theoretical model presented here is related to a pure magnetic response and thus considers the situation when the $5 d$ and $4 f$ spin systems are in equilibrium. The $5 d$ electrons provide a "thermal bath" for the joint magnetization dynamics. The magnetization of the $5 d$ system is neglected and the $4 f$ spin system interacts with external systems (laser and phonons)

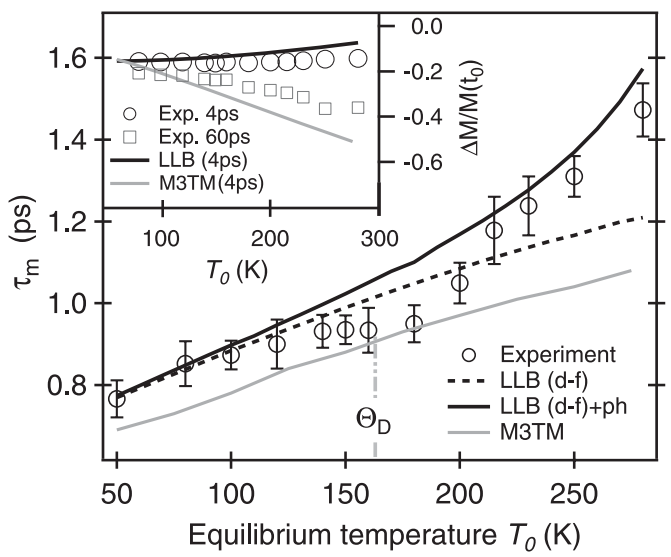

FIG. 4. The demagnetization time $\tau_{m}$ as a function of $T_{0}$. Symbols represent the experimental data points, while lines represent the modeling results considering only electron-mediated spin flips (dashed) and combined electron- and phonon-mediated spin flips (solid line) with $\lambda_{s-p h}^{0}=0.0002$. The gray line represents the results obtained within the M3TM model assuming an EY scattering mechanism. The inset shows $\Delta M / M_{0}$ at the indicated time delays. 
via $5 d$ electrons only. The statistical spin models, such as the classical Heisenberg model ${ }^{3,16,33}$ and the classical LLB model, ${ }^{5,11,17,33}$ have recommended themselves as being able to provide a simple and powerful insight into the dynamics of the ultrafast demagnetization. The spin-flip rate is included in these models as a coupling to the bath parameter, which until now has been considered to be temperature independent. Here we use a multispin quantum LLB model with interlayer heat diffusion and a temperature-dependent coupling to the bath parameter.

Most of the previous works on modeling of ultrafast demagnetization $^{8,11,14,16}$ consider the effect of the electron mechanism only, disregarding the phonon mechanism due to its potentially smaller and slower contribution. Since in Gd the observed demagnetization is slower than in other materials like $\mathrm{Ni}$, the spin-phonon coupling (via the spin-orbit coupling of $5 d$ electrons) can also play an important role. In the following we describe the laser-induced dynamics in Gd by considering that the localized $4 f$ part of its magnetic moment is affected by two contributions: coupling to the $5 d$ fraction through (a) electronic scattering processes and (b) scattering with phonons. We make use of the fact that the phonon population is well characterized as a function of $T_{0}$. The pronounced change in $\tau_{m}\left(T_{0}\right)$ in the vicinity of the Debye temperature $\Theta_{D}=163 \mathrm{~K}$ as depicted in Fig. 4 should provide a key to separate electron- and phonon-mediated spin-flip processes. Consequently, in our model both mechanisms are included. The obtained results are compared to the experimental results in Fig. 4.

The schematic representation of our model for laserinduced demagnetization is shown in Fig. 5. The ultrafast demagnetization dynamics is modelled by a micromagnetic approach based on the (quantum) LLB equation. ${ }^{34,35}$ This equation (without the precessional term) for a system of

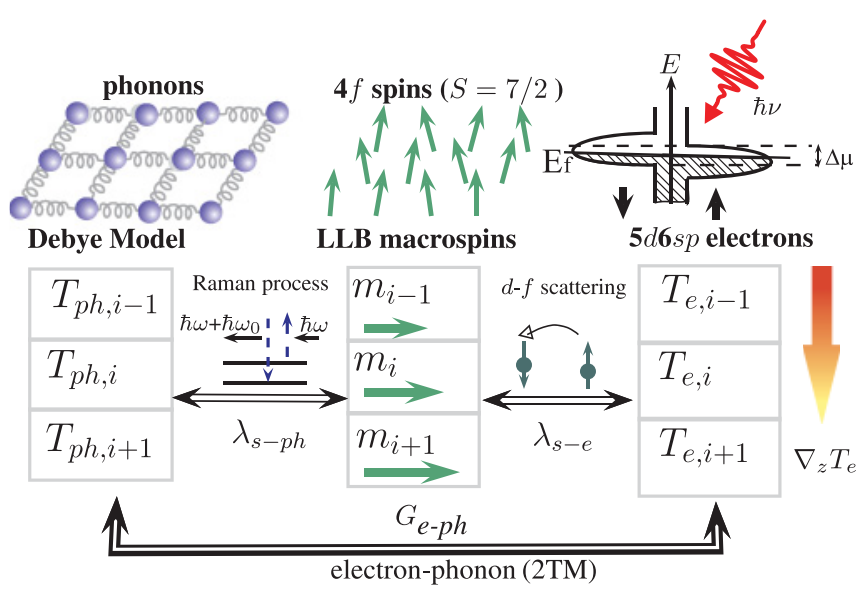

FIG. 5. (Color online) Schematic diagram of the model. The multispin LLB model (center) is coupled to the $2 \mathrm{~T}$ model via two coupling mechanisms: phonon contribution via Raman processes (left) and electron contribution via dynamical spin polarization of carriers which produces a change of chemical potential $\Delta \mu$ (Ref. 37) (right). The 2T model and the LLB model are time and layer resolved and the electron diffusion is considered. The change in the phonon population with temperature is taken into account within the Debye model. The parameters of the $2 \mathrm{~T}$ model were taken from Refs. 27 and 29. ferromagnetic quantum spins interacting with a heat bath describes the dynamics of the thermally averaged spin polarization $m_{i}=\tilde{M}_{i} v_{0} / \mu_{0}$ in the atomic layer $i$ :

$$
\frac{\dot{m}_{i}}{\gamma}=\sum_{\mathrm{b}} \Lambda_{\mathrm{b}}^{i}\left(T_{\mathrm{b}}^{i}\right)\left\{\begin{array}{cc}
\frac{1}{2 \widetilde{\chi}_{\|, \mathrm{b}}^{i}}\left(1-\frac{m_{i}^{2}}{m_{\mathrm{e}, \mathrm{b}, \mathrm{i}}^{2}}\right) m_{i} & T_{\mathrm{b}}^{i} \leqslant T_{\mathrm{C}} \\
-\frac{1}{\widetilde{\chi}_{\|, \mathrm{b}}^{i}}\left(1+\frac{3 m_{i}^{2} T_{\mathrm{C}}}{5\left(T_{\mathrm{b}}^{i}-T_{\mathrm{C}}\right)}\right) m_{i} & T_{\mathrm{b}}^{i} \geqslant T_{\mathrm{C}},
\end{array}\right.
$$

where the sum is over baths $b$ (electron or phonon subsystems). The longitudinal damping parameter $\Lambda_{\mathrm{b}}^{i}\left(T_{\mathrm{b}}^{i}\right)=\lambda_{\mathrm{b}}^{i} \frac{2 T_{\mathrm{b}}^{i}}{3 T_{\mathrm{C}}}\left[\frac{2 q_{\mathrm{b}}^{i}}{\sinh 2 q_{\mathrm{b}}^{i}}\right]$ includes an intrinsic coupling to the bath parameter $\lambda_{\mathrm{b}}^{i}$ (defined by the square of the scattering matrix elements) ${ }^{34}$ as well as the spin part coming from the disordering at the quasiequilibrium bath temperature $T_{\mathrm{b}}^{i}$. Here $\gamma=1.76 \times 10^{11}(\mathrm{~T} \mathrm{~s})^{-1}$ is the absolute value of the gyromagnetic ratio; $m_{e, \mathrm{~b}, i}\left(T_{\mathrm{b}}^{i}\right)$ is the quasiequilibrium magnetization; $q_{\mathrm{b}}^{i}=3 T_{\mathrm{C}} m_{e, \mathrm{~b}, i} /[2(S+$ 1) $\left.T_{\mathrm{b}}^{i}\right] ; v_{0}=(5 \AA)^{3}$ is the unit-cell volume; $\mu_{0}=7.55 \mu_{B}$ is the atomic magnetic moment for $\mathrm{Gd} ; S=7 / 2$ is the spin quantum number; and $T_{\mathrm{C}}=293 \mathrm{~K}$ is the Curie temperature. The longitudinal effective field [r.h.s in Eq. (3)] also accounts for magnetic fluctuations through the longitudinal susceptibility $\tilde{\chi}_{\|, \mathrm{b}}^{i}$ within a dynamical mean-field approach for spin-spin interactions. $^{34}$

In our model for laser-induced demagnetization, the laser excites the $5 d$ electron system which is coupled to the localized $4 f$-electron spin system. After the pump pulse excitation a temperature gradient $\nabla_{z} T_{e}$ is created due to the optical penetration depth $\lambda_{o p}=20 \mathrm{~nm}$ and the electron thermal conductivity, leading to different quasiequilibrium temperatures $T_{e}^{i}(t)$ and $T_{p h}^{i}(t)$ for each layer, obtained from the integration of the 2TM model. ${ }^{27,29}$ We consider the Gd thin film as 40 coupled macrospins, ${ }^{17,33}$ each one representing a 5-Å-thick layer, described by the set of LLB equations [Eq. (3)] and coupled to electron and phonon systems. The exchange coupling between layers is considered to be temperature dependent and scaled with the average magnetization. ${ }^{17,36}$

We focus our attention on the possible dissipation mechanisms leading to a reduction of $\tilde{M}$. For the electronic mechanism, we use the $d$ - $f$ indirect exchange interaction as responsible for exchange of angular momentum between excited $5 d$ carriers and the localized $4 f$ spin system, similar to the $s p$ - $d$ exchange model for magnetic semiconductors. ${ }^{37}$ In this model, the carriers act as a momentum sink and their finite relaxation time $(\sim 100 \mathrm{fs})$ reduces the available phase space for spin-flip events, leading to a dynamical spin polarization of the carriers. In the first approximation we model such a behavior as a time-dependent coupling parameter

$$
\lambda_{s-e}(t)=\lambda_{s-e}^{0}\left[1-\sigma \Delta T_{e}(t) / T_{e}(t)\right] .
$$

The factor $\lambda_{s-e}^{0}$ is adjusted to obtain an experimentally measured demagnetization of $15 \%$ within 4 ps at $T_{0}=50 \mathrm{~K}$, leading to a reasonably small coupling parameter $\lambda_{s-e}^{0}=$ 0.00026 . The value of $\sigma$ is defined by the relaxation time of the carriers. ${ }^{37}$ Therefore, the coupling parameter decreases with the increase of $T_{e}$. The total longitudinal relaxation parameter $\Lambda_{\mathrm{e}}\left(T_{e}\right)$, which includes the spin part, increases with $T_{e}$, but this increase is slowed down for high temperatures. The dashed line in Fig. 4 shows the demagnetization time obtained from the numerical integration of the system of Eq. (3), considering only 
this scattering mechanism. The agreement between modeling (dashed line in Fig. 4) and experimental data holds until $T_{0} \approx 170 \mathrm{~K}$, a value close to $\Theta_{D}=163 \mathrm{~K}$.

Considering the pronounced change in $\partial \tau_{m} / \partial T_{0}$ near $\Theta_{D}$, we add to the LLB equation the spin-phonon relaxation mechanism, coupling the magnetization dynamics also to $T_{p h}$. Note that this coupling is also indirect and occurs through $5 d$ electrons. The Raman process, where a phonon $\mathbf{k}$ is absorbed and a phonon $\mathbf{q}$ is emitted in combination with a spin flip, is adequate to describe the spin-lattice relaxation $\lambda_{s-p h} \sim D^{2} \int d \omega_{\mathbf{k}} \omega_{\mathbf{k}}^{6} n_{\mathbf{k}}\left(n_{\mathbf{k}}+1\right)$, where $D$ is defined by the spin-orbit coupling. ${ }^{20}$ We use the Debye model for the phonon frequency $\omega_{\mathbf{k}}$ for the calculation of $\lambda_{s-p h}$. Its time dependence is defined by $T_{p h}$ in each layer in the following way: ${ }^{20}$

$$
\lambda_{s-p h}(t)=\lambda_{s-p h}^{0}\left(T_{p h} / \Theta_{D}\right)^{7} G_{6}\left(\Theta_{D} / T_{p h}\right),
$$

where $G_{n}(y) \equiv \int_{0}^{y} d x x^{n} e^{x} /\left(e^{x}-1\right)^{2}$. For $T \ll \Theta_{D}$ the rate grows as $T_{p h}^{7}$, whereas for $T \gg \Theta_{D}$ it does as $T_{p h}^{2}$ with a transition between the two regimes at $T_{p h}>0.2 \Theta_{D}$. Thus, the coupling to the phonon subsystem is negligible at $T_{p h}<$ $\Theta_{D}$ but starts to increase with temperature in the vicinity of $\Theta_{D}$. When only the phonon mechanism was considered, the demagnetization time was found in our model to be of the order $\tau_{M} \sim 50-100 \mathrm{ps}$, in agreement with theoretical predictions, ${ }^{20}$ and is found to decrease with temperature. This process alone cannot account for the observed ultrafast demagnetization.

\section{DISCUSSION AND OUTLOOK}

The results of the integration of the LLB equations with the two coupling mechanisms are shown in Fig. 6. The magnetization decay takes place in the time scale up to $50 \mathrm{ps}$ and is not single exponential. Similar to the experiment, we focus our attention to the time scale of several picoseconds and fit the modeling results to single exponential function with a characteristic time scale $\tau_{m}$. The modeling results are in perfect agreement with those presented in Fig. 3. The resulting values of $\tau_{m}$ also show a good quantitative agreement with the experimental data [see Fig. 4 (black line)]. We note that the phonon-mediated demagnetization is intrinsically slower than the electronic one. Thus the observed behavior is different at

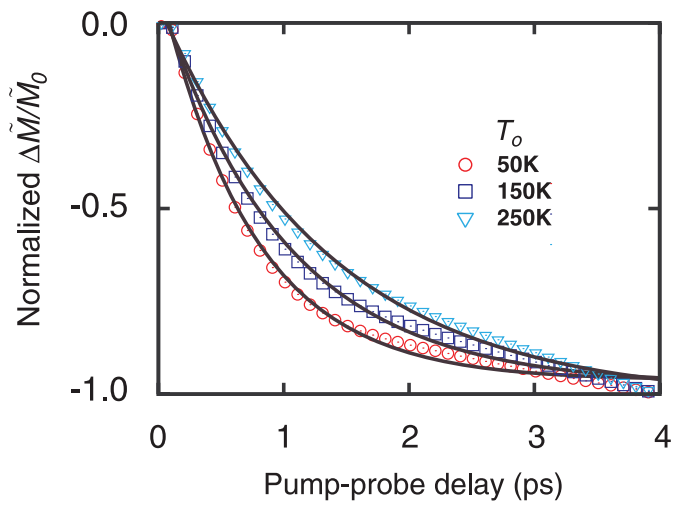

FIG. 6. (Color online) The relative magnetization decay $\Delta \tilde{M} / \tilde{M}_{0}$ for various equilibrium temperatures obtained through the integration of the Landau-Lifshitz-Bloch model (symbols). The line indicates fitting to a single exponential function. different time scales. At $t<4$ ps (neglecting the first 0.2-ps interval) the dynamics is dominated by $T_{e}$. In agreement with the slowing down of $\Lambda_{\mathrm{e}}\left(T_{e}\right)$, the value $\tau_{m}$ increases (indicated in the main panel of Fig. 4) and the experimentally determined demagnetization amplitude $\left|\Delta \tilde{M} / \tilde{M}_{0}\right|(4 \mathrm{ps})$ changes weakly as a function of $T_{0}$ (see inset of Fig. 4). At longer time scales $>10 \mathrm{ps}$, the electron and phonon temperatures have equilibrated and the coupling to the phonon system starts to

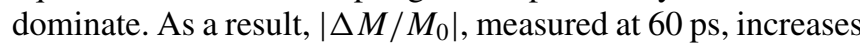
with $T_{0}$ (Fig. 4, inset), contrary to the behavior at 4 ps.

The analysis of experimental and theoretical data also shows that there is a contribution of phonons to $\tau_{m}$ at $T_{0}>\Theta_{D}$, when the coupling to the phonon system increases considerably. The contribution of both relaxation mechanisms decreases $m(t)$ in this time scale and thus additionally slows down the demagnetization via the nonlinearity of the response in Eq. (3).

As the LLB equations correctly take magnetic fluctuations at temperatures close to $T_{\mathrm{C}}$ into account ${ }^{38}$ and explicitly include the critical slowing down effect, we also discard mechanisms based on such fluctuations ${ }^{16}$ as responsible for the observed increase in $\partial \tau_{m} / \partial T_{0}$ for $T_{0} \gtrsim 170 \mathrm{~K}$. Finally, in contrast to the widely used Matthiessen's rule, the scattering rates $\Gamma=1 / \tau_{M}$ of different processes do not add up $\Gamma \neq$ $\Gamma_{s-p h}+\Gamma_{s-e}$ due to the nonlinear dependence of the relaxation rates in Eq. (3) on $m$.

For comparison, we present in Fig. 4 (gray line) the results of the integration of the M3TM model, recently proposed in Ref. 8, which is based on phonon-mediated EY scattering. The M3TM model has been implemented using the same 2T model as the LLB model. It is equivalent to the LLB model with $S=$ $1 / 2$ and $\lambda_{E Y} \sim\left(T_{p h} / T_{e}\right)$ (see Ref. 35). The parameter $\lambda_{E Y}$ has been taken from Ref. 8, which gave a correct demagnetization at 4 ps and $T_{0}=50 \mathrm{~K}$. Although $\tau_{m}$ shows a similar behavior as our LLB results for $T_{0}<170 \mathrm{~K}$ (see Fig. 4), it does not account for the slower demagnetization at $T_{0}>170 \mathrm{~K}$. Furthermore, $\left|\Delta \tilde{M} / \tilde{M}_{0}\right|$ at $4 \mathrm{ps}$ increases with temperature, contrary to the experimental observation.

We would like to mention that in principle a separate equation of motion for the spin polarization of $d$ electrons can be written similar to Ref. 39. In this case we would have to consider that $4 f$ electrons are interacting with the laser pulse and the environment via $5 d$ electrons only. For a macroscopic description of such processes, the derivation of a two-component LLB equation with a correct treatment of $d-f$ exchange interaction within the mean-field approach is necessary. This work is now in progress.

Turning back to the question of possible magnetic contributions to $k_{r, e} / k_{0 r, e}$, we suggest here a scenario for a consistent explanation of the few hundred femtoseconds regime, which is still speculative. In $\mathrm{Gd}$, the direct coupling of $4 f$ spins to the lattice is inhibited by the zero orbital momentum of the $4 f$ shell and only the $5 d$ electrons present the spin-orbit interaction required for an angular momentum transfer to the lattice. Due to that, the angular momentum is transferred (i) from $4 f$ magnetic moments to $5 d$ spins and then (ii) from $5 d$ spins to the lattice. The efficiency of the last step could be limited by the available phonons forming a phonon bottleneck for demagnetization. This effect would be even larger at early delays when only few phonons are 
excited. Finally, we can suppose that the process (ii) is faster for higher equilibrium temperature $T_{0}$ and accelerates with the increasing pump-probe delay $t$. On the contrary, process (i) may be accelerated by the elevated electron temperature, ${ }^{40}$ i.e., it may slow down with the increasing $t$. Such variations of relative spin transfer rates of the two processes could lead to the angular momentum accumulation in the spin subsystem of $5 d$ electrons at low $T_{0}$ and small $t$ if the channel (i) is fast enough, which is the case for Gd where the energy of the intra-atomic-like exchange interaction between $4 f$ magnetic moments and spins of $5 d$ electrons is about $700 \mathrm{meV}^{41}$ This transient increase of the conduction-band spin polarization which primarily influences the MOKE signal is in agreement with our observation in Figs. 1 and 2 at $50 \mathrm{~K}$ within $t<300 \mathrm{fs}$. The verification of this scenario and more detailed analysis of the spin dynamics proceeding before the equilibration of $5 d$ spins and $4 f$ magnetic moments would require further experimental and theoretical efforts, which hopefully will be stimulated by our observations.

\section{CONCLUSION}

We performed a temperature-dependent analysis of femtosecond-laser-induced demagnetization of $\mathrm{Gd}(0001)$ by time-resolved MOKE and the Landau-Lifshitz-Bloch model. We separate the obtained experimental data into two regimes: (i) several $100 \mathrm{fs}$ after the optical excitation, when the $5 d$ conduction electrons carry the major part of the excess energy, and (ii) a subsequent regime until several picoseconds, i.e., the regime in which the conduction electrons equilibrate with the phonons. We chose this two-component analysis because we recognize in the second regime a single exponential variation of the transient magnetization which was in previous work assigned to magnetization dynamics of the $4 f$ magnetic moment.

In the picosecond regime we find a transient variation of the MOKE ellipticity and rotation which agrees well with each other and we assign them to the magnetization dynamics dominated by the $4 f$ magnetic moment. Here, we observe a 2 times increase in the characteristic demagnetization time from $0.8 \mathrm{ps}$ at $50 \mathrm{~K}$ to $1.5 \mathrm{ps}$ at $280 \mathrm{~K}$ which we explain on the basis of the results from the Landau-Lifshitz-Bloch model by microscopic electron- and phonon-mediated demagnetization processes quantitatively. In general, a temperature increase results in a slower demagnetization. At low temperature the demagnetization times observed in the experiment can be modelled by considering electronic processes only. At temperatures above the Debye temperature, phonon-mediated processes have to be taken into account in addition.

We also find indications of processes at time scales faster than the above demagnetization, which we assign to $5 d$ electrons. Albeit the temperature- and time-dependent MOKE ellipticity and rotation data exhibit a more complex behavior, we consider that these indications of magnetic and nonmagnetic contributions are beyond the above-mentioned demagnetization process. In general, we expect that understanding this very early spin-dependent dynamics sheds light on the dynamic and microscopic nature of the spin-dependent interplay of $4 f$ and $5 d$ electrons.

\section{ACKNOWLEDGMENTS}

We gratefully acknowledge funding by the DAAD-HEC Pakistan, the DFG through ME-3570/1, the BMBF through FEMTOSPEX, and the Spanish Ministry of Science and Innovation under Grant No. FIS2010-20979-C02-02. *uwe.bovensiepen@uni-due.de

${ }^{1}$ A. Kirilyuk, A. V. Kimel, and T. Rasing, Rev. Mod. Phys. 82, 2731 (2010).

${ }^{2}$ G. Ju, J. Hohlfeld, B. Bergman, R. J. M. van de Veerdonk, O. N. Mryasov, J.-Y. Kim, X. Wu, D. Weller, and B. Koopmans, Phys. Rev. Lett. 93, 197403 (2004).

${ }^{3}$ I. Radu, K. Vahaplar, C. Stamm, T. Kachel, N. Pontius, H. A. Dürr, T. A. Ostler, J. Barker, R. F. L. Evans, R. W. Chantrell et al., Nature (London) 472, 205 (2011).

${ }^{4}$ C. D. Stanciu, F. Hansteen, A. V. Kimel, A. Kirilyuk, A. Tsukamoto, A. Itoh, and T. Rasing, Phys. Rev. Lett. 99, 047601 (2007).

${ }^{5}$ K. Vahaplar, A. M. Kalashnikova, A. V. Kimel, D. Hinzke, U. Nowak, R. Chantrell, A. Tsukamoto, A. Itoh, A. Kirilyuk, and T. Rasing, Phys. Rev. Lett. 103, 117201 (2009).

${ }^{6}$ A. Weber, F. Pressacco, S. Günther, E. Mancini, P. M. Oppeneer, and C. H. Back, Phys. Rev. B 84, 132412 (2011).

${ }^{7}$ S. Essert and H. C. Schneider, Phys. Rev. B 84, 224405 (2011).

${ }^{8}$ B. Koopmans, G. Malinowski, F. Dalla, D. Steiauf, M. Fähnle, T. Roth, M. Cinchetti, and M. Aeschlimann, Nature Mater. 9, 259 (2010).

${ }^{9}$ J. Walowski, G. Müller, M. Djordjevic, M. Münzenberg, M. Kläui, C. A. F. Vaz, and J. A. C. Bland, Phys. Rev. Lett. 101, 237401 (2008).
${ }^{10}$ M. Pickel, A. B. Schmidt, F. Giesen, J. Braun, J. Minár, H. Ebert, M. Donath, and M. Weinelt, Phys. Rev. Lett. 101, 066402 (2008).

${ }^{11}$ U. Atxitia, O. Chubykalo-Fesenko, J. Walowski, A. Mann, and M. Münzenberg, Phys. Rev. B 81, 174401 (2010).

${ }^{12}$ A. B. Schmidt, M. Pickel, M. Donath, P. Buczek, A. Ernst, V. P. Zhukov, P. M. Echenique, L. M. Sandratskii, E. V. Chulkov, and M. Weinelt, Phys. Rev. Lett. 105, 197401 (2010).

${ }^{13}$ M. Krauß, T. Roth, S. Alebrand, D. Steil, M. Cinchetti, M. Aeschlimann, and H. C. Schneider, Phys. Rev. B 80, 180407 (2009).

${ }^{14}$ M. Battiato, K. Carva, and P. M. Oppeneer, Phys. Rev. Lett. 105, 027203 (2010).

${ }^{15}$ A. Melnikov, I. Razdolski, T. O. Wehling, E. T. Papaioannou, V. Roddatis, P. Fumagalli, O. Aktsipetrov, A. I. Lichtenstein, and U. Bovensiepen, Phys. Rev. Lett. 107, 076601 (2011).

${ }^{16}$ N. Kazantseva, D. Hinzke, R. W. Chantrell, and U. Nowak, Europhys. Lett. 86, 27006 (2009).

${ }^{17}$ U. Atxitia, O. Chubykalo-Fesenko, N. Kazantseva, D. Hinzke, U. Nowak, and R. W. Chantrell, Appl. Phys. Lett. 91, 232507 (2007).

${ }^{18}$ A. Vaterlaus, T. Beutler, and F. Meier, Phys. Rev. Lett. 67, 3314 (1991). 
${ }^{19}$ M. Wietstruk, A. Melnikov, C. Stamm, T. Kachel, N. Pontius, M. Sultan, C. Gahl, M. Weinelt, H. A. Dürr, and U. Bovensiepen, Phys. Rev. Lett. 106, 127401 (2011).

${ }^{20}$ W. Hübner and K. H. Bennemann, Phys. Rev. B 53, 3422 (1996).

${ }^{21}$ K. A. Gschneidner and J. L. Eyring, Handbook on the Physics and Chemistry of Rare Earths (Elsevier, North-Holland, 1997), Vol. 24.

${ }^{22}$ P. Kurz, G. Bihlmayer, and S. Blügel, J. Phys.: Condens. Matter 14, 6353 (2002).

${ }^{23}$ B. Koopmans, M. van Kampen, J. T. Kohlhepp, and W. J. M. de Jonge, Phys. Rev. Lett. 85, 844 (2000).

${ }^{24}$ L. Guidoni, E. Beaurepaire, and J.-Y. Bigot, Phys. Rev. Lett. 89, 017401 (2002).

${ }^{25}$ P. M. Oppeneer and A. Liebsch, J. Phys.: Condens. Matter 16, 5519 (2004).

${ }^{26}$ A. Aspelmeier, F. Gerhardter, and K. Baberschke, J. Magn. Magn. Mater. 132, 22 (1994).

${ }^{27}$ U. Bovensiepen, J. Phys.: Condens. Matter 19, 083201 (2007).

${ }^{28}$ M. Sultan, A. Melnikov, and U. Bovensiepen, Phys. Status Solidi B 248, 2323 (2011).

${ }^{29} \mathrm{M}$. Lisowski, $\mathrm{PhD}$ thesis, Freie University Berlin (2005); http://www.diss.fu-berlin.de/diss/receive/FUDISS_ thesis_000000001938.
${ }^{30}$ S. I. Anisimov, B. L. Kapeliovich, and T. L. Perel'man, Sov. Phys. JETP 39, 375 (1974).

${ }^{31}$ W. Nolting, T. Dambeck, and G. Borstel, Z. Phys. B 94, 409 (1994).

${ }^{32}$ K. Maiti, M. C. Malagoli, A. Dallmeyer, and C. Carbone, Phys. Rev. Lett. 88, 167205 (2002).

${ }^{33}$ N. Kazantseva, D. Hinzke, U. Nowak, R. W. Chantrell, U. Atxitia, and O. Chubykalo-Fesenko, Phys. Rev. B 77, 184428 (2008).

${ }^{34}$ D. A. Garanin, Physica A 172, 470 (1991).

${ }^{35}$ U. Atxitia and O. Chubykalo-Fesenko, Phys. Rev. B 84, 144414 (2011).

${ }^{36}$ P. Asselin, R. F. L. Evans, J. Barker, R. W. Chantrell, R. Yanes, O. Chubykalo-Fesenko, D. Hinzke, and U. Nowak, Phys. Rev. B 82, 054415 (2010).

${ }^{37}$ L. Cywiński and L. J. Sham, Phys. Rev. B 76, 045205 (2007).

${ }^{38}$ O. Chubykalo-Fesenko, U. Nowak, R. W. Chantrell, and D. Garanin, Phys. Rev. B 74, 094436 (2006).

${ }^{39}$ J. Seib and M. Fähnle, Phys. Rev. B 82, 064401 (2010).

${ }^{40} \mathrm{~T}$. Wehling, M. Katsnelson, and A. Lichtenstein (private conversations).

${ }^{41}$ R. Ahuja, S. Auluck, B. Johansson, and M. S. S. Brooks, Phys. Rev. B 50, 5147 (1994). 